\title{
A Scalable Movie Recommendation System on Search Engine
}

\author{
Mingruo Shi \\ Beijing Wuzi University, 101149, China \\ shimingruo@163.com
}

Keywords: Recommendation, Content based filtering, Ranking, Hadoop.

\begin{abstract}
A recommendation system is increasing important in online video application, news recommendation and electronic commerce especially in search engine. Recommendation on search engine are more related to hundreds of million users like Google, Bing and Baidu. Our proposed filtering solution is based on content match to handle large number of users and movies for search engine.
\end{abstract}

\section{Introduction}

Recommender systems have become extremely common in recent years, and are utilized in a variety of areas: some popular applications include movies, music, news, books, research articles, search queries, social tags, and products in general. There are also recommender systems for experts [1], collaborators [2], jokes, restaurants, garments, financial services [3], life insurance, online dating, and Twitter pages [4].

Recommender systems typically produce a list of recommendations in one of two ways - through collaborative and content-based filtering or the personality-based approach [5, 11, 12]. Collaborative filtering approaches building a model from a user's past behavior (items previously purchased or selected and/or numerical ratings given to those items) as well as similar decisions made by other users. This model is then used to predict items (or ratings for items) that the user may have an interest in [6]. Content-based filtering approaches utilize a series of discrete characteristics of an item to recommend additional items with similar properties [7]. These approaches are often combined (called Hybrid Recommender Systems).

A recommendation system usually including two major parts. One is recommendation which will create a recommendation item candidates based on user's visit/click history. The other part is to create top $\mathrm{n}$ recommendation results by ranking the recommended candidates. There are some papers discussing some scalable recommendation to users including Google news recommendation [8,10]. To our best knowledge, no literature gives the detailed introduction how a large-scale recommendation system is designed and implemented scaled both to number of users and items on the movie recommendation vertical.

In this paper, we proposed a Content-based filtering recommendation algorithm scalable to both users and items which can be applied to movie recommendation esp. on search engine.

The paper is organized as follows: in section 2, we describe in detail our algorithm to support large number of users and movies. In section 3 introduces our experiments. We summarize our ongoing work in section 4.

\section{Algorithm}

\subsection{Problem Statement.}

Problem statement is for recommendation as follows:

Input:

A list of $\mathrm{n}$ users $\left\{U_{1}, U_{2}, \ldots, U_{n}\right\}$ with user profile

A list of m movies $\left\{M_{1}, M_{2}, \ldots, M_{m}\right\}$ with movie content and status information

The visit history of all users for the fixed period (e.g. 3-month log)

Output: 
A list of preferred movies for each user for today.

Our solution is to develop a content-based collaborative filtering recommendation system. We firstly extract the side information for user to build user profile and movie information for match. Then use the visiting history and side information including user profile and movie information in both training and rank phases of the algorithm.

\subsection{Basic Idea.}

At high level, the idea to recommend to a user, say $A$, is to find the set of users, say $\mathcal{C}_{A}$, that are like $A$, and recommend to $A$ items viewed by users in $\mathcal{C}_{A}$. Pseudocode:

Algorithm A:

Choose a threshold $\theta$
For each user $\mathrm{A}$ and possible interested movie $\mathrm{M}$
$\bullet \quad \mathrm{C}_{\mathrm{A}} \leftarrow \Phi / /$ the set of movies user A may be interested in
- If $\mathrm{s}\left(\mathrm{P}_{\mathrm{A}}, \mathrm{M}\right) \geq \mathrm{Q} / / \mathrm{S}\left(\mathrm{P}_{\mathrm{A}}, \mathrm{M}\right)$ is the score how A is possibly interested in movie $\mathrm{M}$
Add $\left(\mathrm{M}, \mathrm{s}\left(\mathrm{P}_{\mathrm{A}}, \mathrm{M}\right)\right)$ to $\mathrm{C}_{\mathrm{A}}$
- Recommend to A at most k movies in $\mathrm{C}_{\mathrm{A}}$ by rank score $\mathrm{s}\left(\mathrm{P}_{\mathrm{A}}, \mathrm{M}\right)$

\subsection{Build User Profile.}

Nowadays search engine has the capacity to build user profile based on user's search behavior. The basic user profile information includes age, location, gender etc. which could get for registered users.

Besides the mentioned basic user information, search engine could leverage the information inferred from users daily search activities i.e. searched queries and click activities. For movie segment, barely queries themselves are hard to build user profile.

We build user profile by leveraging search graph of movie entity detailed information. The most important information will be extracted from movie entity are title, country, genre, director, actors, actresses, release date, on theater state and ratings. Then we build (feature, feature value) key value pairs by aggregating a fixed period search history for example 90 days.

Movie features for user profiles could be built by different ways for a specific recommendation target. Propose one way including features: country, genre, director, actor and release date. The feature value is percentage by aggregating last 90 -day search history.

\subsection{Extract Movie Features.}

Our target is to recommend movies to users. We therefore only consider active listing movies. And extract the same features as user profile. For example, we could extract features such as title, country, genre, director, actors, actresses, release date, on theater state and ratings.

\subsection{Rank Recommendation Result.}

We could calculate match score between users and movies with equal country and city. For matched user and movie pair, we could get match score by the following way:

$$
s_{u, m}=\sum_{i=1}^{n} c_{i} f_{i} \text {. }
$$

$s_{u, h}$ Is the match score. $\mathrm{n}$ is the number of matched features for user $\mathrm{u}$ and movie $\mathrm{h} . f_{i}$ is the feature value in user's profile for a give user $\mathrm{u}$, we get top k movies ranked by match score satisfy $s_{u, h_{1}} \geq$ $s_{u, h_{2}} \geq \cdots \geq s_{u, h_{k}}$ where $\mathrm{k}$ is a predefined number.

\subsection{Decide Coefficients.}

There are a few ways to decide the coefficients $c_{i}$ during user and movie matching process. We use logistic regression machine learning method on user search history to figure out the optimized coefficients $c_{i}$.

Finally search engine could make a recommendation to user in some ways. For example, a user could be recommended by search engine's home page or recommendation email. 


\section{Experiments}

\subsection{Evaluation Methodology.}

We take three-month movie entity search log in our test search engines and split the visit log into the training set and the test set. The test set is one-day log, i.e., the visit log of the latest day in the $\log$. We use the training set to get recommendation for every user and compare the recommendation list with the list of movies that users visited in the test set. When we measure, we remove movies in the test file that is NOT in the training file. Here note that the offline evaluation does not truly reflect the performance of the online system (the performance should be much better when we launch the system online) since we haven't posed the recommendation to users yet. But it helps us determine what ideas can potentially improve the performance of the online system.

Running time: In our log, the number of distinct users is up to 13292550 ( 13 million) and movies is 1429482 (about 1 million). The training can be done in 5 hours, using about 100 computers in a Hadoop cluster. So, in the following, we mainly discuss about the prediction accuracy.

Two main metrics below are considered for the prediction accuracy:

(1) TrueRate: We assign score 1 to a user if he/she visited a recommended item in the test set and 0 otherwise.

TrueRate $=$ Total Score $/($ Total \# Users $)$.

Essentially, TrueRate computes the fraction of users searched for at least one recommendation in the test set

(2) NDCG (Normalized Discounted Cumulative Gain): we define NDCG @ k as:

$$
N D C G_{k}=\frac{D C G_{k}}{D C G_{k}^{*}}
$$

Where $D C G_{k}=\sum_{i=1}^{k} \frac{2^{r e l_{i}}-1}{\log _{2}(i+1)}$ and $D C G_{k}^{*}$ is the ideal$D C G_{k}$. We assign $r e l_{i}$ to equal 1 if there was a click on item ranked in positioni. Here, $k$ is the recommendation length, i.e, select top $k$ movies to present to users. Basically, NDCG assign higher score to visited items that in the top of the recommendation list than the visited items in the bottom of the list.

\subsection{Evaluation Result.}

Evaluation Result Besides our algorithm, we also developed a Collaborative Filtering algorithm which doesn't use any movie entity information at all. However, our algorithm takes important signals from users' search history and search graph. Note that we only take top (at most) 3 movies to recommend to users. The evaluation results are shown in Fig. 1 and Fig. 2.

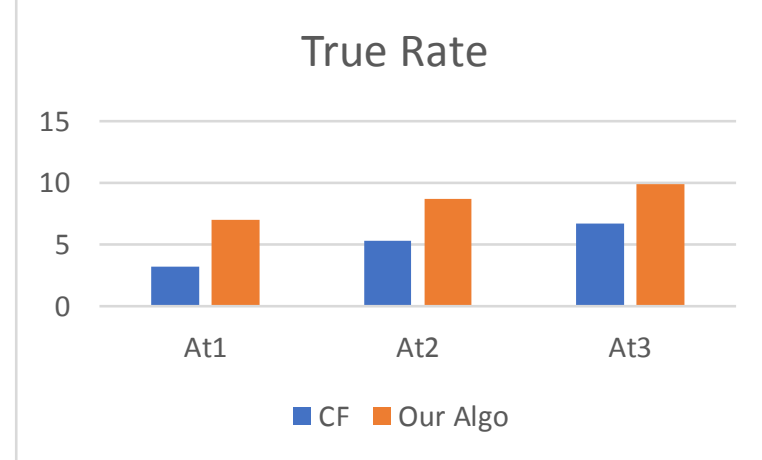

Fig. 1 True Rate

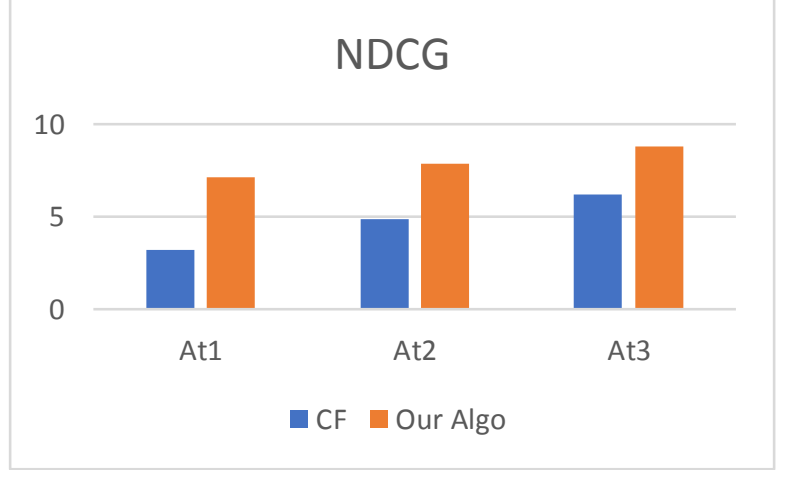

Fig. 2 NDCG

As Fig. 1 and Fig. 2 suggest, our algorithm clearly outperforms collaborative filtering algorithm.

\section{Future Work}

Firstly, personalized ranking methods from Information Retrieval could be leveraged. Researchers came up with various ranking strategies to present relevant information to users. There is a line of research that incorporate user's interests into the ranking, called personalized ranking [11]. The same idea can be applied in ranking the recommendation result. Finally, probabilistic recommendation 
algorithm might be helpful. pLSI (probabilistic latent semantic indexing)-based algorithm may outperform our hashing-based algorithm [12].

\section{Acknowledgements}

This research was financially sponsored by the key lab of intelligent logistic system BZ0211 PXM2015_014214_00000.

\section{References}

[1]. H. Chen, A. G. Ororbia II, C. L. Giles ExpertSeer: a Keyphrase Based Expert Recommender for Digital Libraries, in arXiv preprint 2015.

[2]. H. Chen, L. Gou, X. Zhang, C. Giles Collabseer: a search engine for collaboration discovery, in ACM/IEEE Joint Conference on Digital Libraries (JCDL) 2011

[3]. Alexander Felfernig, Klaus Isak, Kalman Szabo, Peter Zachar, The VITA Financial Services Sales Support Environment, in AAAI/IAAI 2007, pp. 1692-1699, Vancouver, Canada, 2007.

[4]. Pankaj Gupta, Ashish Goel, Jimmy Lin, Aneesh Sharma, Dong Wang, and Reza Bosagh Zadeh WTF:The who-to-follow system at Twitter, Proceedings of the 22nd international conference on World Wide Web.

[5]. Hosein Jafarkarimi; A.T.H. Sim and R. Saadatdoost A Naïve Recommendation Model for Large Databases, International Journal of Information and Education Technology, June 2012

[6]. Prem Melville and Vikas Sindhwani, Recommender Systems, Encyclopedia of Machine Learning, 2010.

[7]. R. J. Mooney \& L. Roy (1999). Content-based book recommendation using learning for text categorization. In Workshop Recom. Sys.: Algo. and Evaluation.

[8]. Das, Abhinandan S., et al. "Google news personalization: scalable online collaborative filtering." WWW'07.

[9]. Indyk, Piotr. "A small approximately min-wise independent family of hash functions." Journal of Algorithms 38.1 (2001): 84-90.

[10]. Jure Leskovec, Anand Rajaraman, Jeff Ullman, "Mining of Massive Datasets", Chapter 3.

[11]. Bouadjenek, Mohamed Reda, et al. "Evaluation of personalized social ranking functions of information retrieval." ICWE'13

[12]. Liu, Jiahui, et al. "Personalized news recommendation based on click behavior." ICIUI' 10. 